\title{
CAN WE MANAGE NEONATAL INFECTION IN THE COMMUNITY ?
}

\author{
Manandhar D S , Costello A M*, Osrin D*
}

\section{ABSTRACT}

As infant mortality rates have fallen in many developing countries, the problem of neonatal mortality has become more obvious. The biggest causes of mortality in the first month of life are infection, birth asphyxia, and low birth weight. Infection is implicated in about a third of neonatal deaths in Nepal. Communitybased data are limited, but neonatal sepsis is likely to be the result of infection by Gram positive bacteria such as Staphylococci and Streptococci, and enteric Gram negatives.

The appropriate management for neonatal sepsis is parenteral, hospital-based treatment with a penicillin and an aminoglycoside. However, about $90 \%$ of births in Nepal take place at home, and many infants never reach hospital. For these infants, the next best management strategy is to give parenteral antibiotics at a primary care facility. Before referral, it would be appropriate to give a dose of oral antibiotic such as cotrimoxazole, which is already incorporated into the acute respiratory infection programme. If referral for parenteral treatment is not successful, we propose that community-based cadres be allowed to give a full course of oral antibiotic in cases of neonatal sepsis.

Community health workers should receive training and pictorial guidelines for the recognition of danger signs for neonatal sepsis, and we recommend pilot studies to compare and evaluate oral treatment in the community. For Nepal, a national policy on the community management of neonatal infection is an extremely urgent priority.

Key Words: Neonatal infection, community management, antibiotic use.

\section{INTRODUCTION}

Infant mortality rates have fallen steadily in many developing countries over the last two decades. Unfortunately, there has been a tendency for the decline to level off. ${ }^{1}$ This appears to be because of the relatively resistant contribution of neonatal mortality. While postneonatal mortality rates have fallen sharply, neonatal mortality rates have remained fairly static, ${ }^{2}$ and now account for about two-thirds of infant mortality as a whole. ${ }^{3}$ If we are to capitalise on the gains made in Acute Respiratory Infection (ARI), Control of Diarrhoeal Disease (CDD) and the Expanded Programme of Immunisation (EPI) by His Majesty's Government, Nepal (HMG/N), we will need to focus on the neonatal period. The most important causes of mortality in the first month of life are infection, neonatal encephalopathy caused by presumptive birth asphyxia, and low birth weight (especially the result of preterm delivery). ${ }^{4}$

\footnotetext{
* Kathmandu Medical College, GPO Box: 921, Kathmandu, Nepal.

** International Perinatal Care Unit, Institute of Child Health, University College London, 30 Guilford Street, London WC1N 1EH.

Address for correspondence : $\quad$ Prof. D. S. Manandhar

Department of Paediatrics

Kathmandu Medical College, Kathmandu, Nepal.

Email: dsm@healthnet.org.np
} 


\section{Infection as a cause of neonatal mortality}

The contribution of infection to neonatal mortality in Nepal is definitely substantial but inadequately documented. Work from a rural programme in Gadchiroli, Maharashtra, India suggests that $17 \%$ of neonates born in villages develop clinical features suggestive of sepsis. ${ }^{5}$ Although the information on causespecific neonatal mortality is limited, several studies have been carried out. A community-based prospective study in Lahore, Pakistan found that infection - in the form of septicaemia, pneumonia and meningitis - contributed to $31 \%$ of neonatal deaths. ${ }^{6}$ Tetanus neonatorum was responsible for a further seven percent. Surveillance findings from Matlab, Bangladesh for the period 1989-90 suggest that infections account for $23 \%$ of neonatal deaths. ${ }^{7}$ Structured verbal autopsy in 287 neonatal deaths identified by the Bangladesh Demographic and Health Survey ascribes $29 \%$ of them to infection. ${ }^{8}$ Finally, WHO estimates quoted in the State of the World's Newborns suggest that infections (tetanus, sepsis, pneumonia, and diarrhoea) account for $32 \%$ of neonatal mortality. ${ }^{3}$ It seems likely, therefore, that infection is implicated in between a quarter and a third of neonatal deaths in rural Nepal.

\section{Pathogenic bacteria}

If we have limited data on cause-specific mortality, we have even less on the microbiology of neonatal sepsis in the community. The pathogens found in studies carried out in hospitals may be entirely different from those causing disease in Nepal's villages, particularly in terms of nosocomial pathogens such as methicillin-resistant Staphylococcus aureus. ${ }^{9}$

One attempt to answer the question of microbiological cause was made by the WHO multicentre study of serious infection in young infants. The study was conducted in four centres over a period of two years, and looked at blood and cerebrospinal fluid cultures in infants under the age of three months. In a children's hospital in Addis Ababa, Ethiopia, the commonest isolates were Streptococcus pneumoniae (24\%), Streptococcus pyogenes (22\%) and Salmonella group B $(12 \%) .{ }^{10}$ In a hospital in Alabang, Philippines, the commonest isolates were Gram negative organisms (71\% of isolates), especially Salmonella species, Enterobacter species and Escherichia coli. Staphylococcus aureus, Streptococcus pneumoniae and Streptococcus pyogenes accounted for $9 \% .{ }^{11}$ At a base hospital in the Highlands of Papua New Guinea, Streptococcus pyogenes and Staphylococcus aureus were the most important pathogens isolated from children under one month old with severe infections. ${ }^{12}$ And in The Gambia the most important causes of serious infections in young infants were Staphylococcus aureus, Streptococcus pneumoniae and Gram negatives including Salmonella species and Escherichia coli. ${ }^{13}$ A recent study from Papua New Guinea looked at consecutive childhood deaths in a Highland hospital and concluded that the mortality burden from sepsis due to enteric Gram negative bacilli was large relative to its overall incidence. ${ }^{14}$ Of 126 neonatal deaths, infection was implicated in $66 \%$. Putting aside the fact that congenital syphilis was implicated in $13.5 \%$, and that the hospital probably saw a population biased toward sepsis, gram negative organisms (such as Pseudomonas species, Klebsiella species, and Escherichia coli) were isolated in about $22 \%$, and gram positive organisms in $2 \%$.

In summary, although community based data are limited, it seems reasonable to suggest that neonatal sepsis is likely to be the result of infection by Staphylococcus aureus, Streptococcus pneumoniae and Gram negative organisms such as Salmonella, Escherichia coli, Klebsiella species and Enterobacter species.

\section{Potential treatments}

So far, we have discussed the burden and microbiology of neonatal infection in rural Nepal. Let us be clear that we know which treatments are likely to be effective: combinations of a penicillin (usually penicillin or ampicillin) and an aminoglycoside (gentamicin or amikacin) are most appropriate, and need to be given parenterally. However, for the sake of discussion, the likely sensitivities of the bacteria discussed above to a number of oral antibiotics are summarised in the table. We do not have information on the treatment of neonatal sepsis with oral antibiotics: the literature is hospital based and treatment is always parenteral. The Gadchiroli project achieved a fall in neonatal mortality of over $30 \%$ through community treatment of sepsis by village health workers, although the treatment itself involved a combination of oral cotrimoxazole with intramuscular gentamicin. ${ }^{15}$

\section{HOW SHOULD A COMMUNITY-BASED HEALTH WORKER MANAGE NEONATAL SEPSIS?}

\section{Hospital treatment is best}

Over $90 \%$ of births in Nepal take place at home, ${ }^{16}$ and treatment of most neonatal problems will of necessity take place at community level, either in the home or at Sub-Health Posts, Health Posts or Primary Health Centres. The health worker is likely to encounter neonatal sepsis at one of these points. What should the health worker do? $\mathrm{HMG} / \mathrm{N}$ has protocolised the management of acute respiratory infection in children, and as such this may stand as a model for the management of sepsis, particularly since ARI constitutes a substantial part of sepsis overall. The control of ARI is summarised in two manuals, ${ }^{17,18}$ and is particularly addressed to community based workers: “. .. the programme will focus on strengthening the community based approach through emphasizing the role of the Village Health Workers (VHWs), Female Community Health Volunteers (FCHVs), and mothers...". ${ }^{17}$ 
The key point to note is that the protocols are split by age group. Above the age of two months, the standard management protocol for ARI (the mainstay of which is oral cotrimoxazole as first line) apply. Below the age of two months, ARI is classified as follows: (1) Very severe disease, (2) Severe pneumonia, and (3) No pneumonia. ${ }^{18}$ Note that any pneumonia in this age group is considered to be severe. We echo the HMG/N advice that any young infant with pneumonia is at high risk and should be hospitalized if at all possible. We may extend this to include all types of sepsis: pneumonia, meningitis and septicaemia. Clearly, the correct treatment for sepsis in this age group is a hospital based combination of penicillin and aminoglycoside, given parenterally. However, here we run into a problem as majority of the sick neonates may not reach a hospital.

\section{What to do for the infants who do not reach a hospital?}

Supposing that geographical, family and financial considerations mean that a family with a sick infant do not take her to an appropriate hospital. We know from health service utilisation and care-seeking behaviour studies in Nepal that most sick infants do not reach a hospital. How is a community based health worker to proceed? First, we would again counsel that referral for intensive treatment with parenteral antibiotics, and possibly for intravenous hydration, is the correct treatment. HMG/N points out that “... past experience has shown that the difficult, or lack of, accessibility of populations to health facilities has been a major factor that has led to high ARI mortality, especially among young infants who may die when essential and timely treatment is not available... ". ${ }^{17}$ The next best management, therefore, would be to give parenteral antibiotics at a primary care facility, and staff and facilities for this should be available. Before referral, it is appropriate to give some form of treatment, and the ARI guidelines suggest that the community based worker "... Refer urgently to hospital if at all possible after giving first dose of Cotrimoxazole...". ${ }^{18}$

\section{What about treatment at home?}

However, there is still the possibility that, because of distance, economics, staff shortages or other reasons, home treatment is preferred by the family. In this case, “... in selected areas and in situations ... Village Health Workers (VHWs), Female Community Health Volunteers (FCHVs), and other community health workers may be trained in the Standard Case Management of Pneumonia and provided with first line drugs... ", ${ }^{18}$ and may "... If referral is not possible due to distance or lack of parents' compliance, treat with a full course of Cotrimoxazole for 5 days...". ${ }^{18}$

We endorse these recommendations as eminently sensible, and summarise the discussion as follows. Since sepsis accounts for around $30 \%$ of neonatal mortality, its identification and treatment are a priority. Treatment in Hospital or PHC with parenteral penicillin and aminoglycoside is the counsel of perfection. At the point of referral, it seems reasonable to give a dose of oral antibiotic (cotrimoxazole would be easiest, as it is cheap, available, part of the ARI control programme, and has a reasonably appropriate spectrum as discussed above). If referral for parenteral treatment is not successful for any reason, we propose that community based cadres be allowed to give a full course of oral antibiotic in cases of neonatal sepsis. It seems to us that in this case the alternative to oral treatment is no treatment at all.

\section{Other questions}

The discussion so far has focussed on the management of neonatal sepsis. This depends, however, on its recognition. 